\title{
MOBILIZANDO O RACIOCÍNIO PROPORCIONAL NA RESOLUÇÃO DE QUESTÕES PERCENTUAIS VIA USO DE FUNÇÕES LINEARES
}

\author{
Paulo Jorge Magalhães Teixeira (D) 1
}

\section{Resumo}

Este trabalho tem o objetivo de mostrar resultados e análises de dados das 3 últimas questões de uma avaliação, que contou com um total de 6 questões (as 3 primeiras questões foram objeto de análise em outro artigo). A avaliação foi proposta em sala de aula de um colégio público federal localizado em uma grande cidade, capital de estado, para alunos de uma turma da $1^{\text {a }}$ série do ensino médio e foi entregue por 12 alunos (houve 10 faltas, e 6 alunos não a entregaram). O propósito que norteou o estudo foi descrever a natureza das soluções dos sujeitos - conforme o nível de mobilização do raciocínio proporcional em relação à determinação de aumentos e de decréscimos percentuais de valores e quantias - de modo a conhecer e identificar como se deu a natureza das intervenções de ensino encaminhadas pelo professor. A avaliação deu-se na aula seguinte ao término do desenvolvimento e exploração de conceitos associados ao exercício do raciocínio proporcional via conceitos pertinentes às funções lineares, durante reflexões feitas quando da resolução de questões de Matemática Financeira, ao longo de 4 aulas, duração de 40 minutos cada. Trata-se de uma pesquisa qualitativa, na modalidade pesquisa-ação estratégica, segundo a qual a análise dos dados constituiu o mote para apontar indicações a respeito da apropriação do raciocínio proporcional. Os resultados mostraram que a metodologia e os procedimentos de resolução desenvolvidos para este universo de situações pouco atraíram os sujeitos de estudo como uma alternativa de resolução.

Palavras-chave: Proporcionalidade; Porcentagem; Raciocínio Proporcional; Funções Lineares.

\section{MOBILIZING PROPORTIONAL REASONING IN SOLVING PERCENTAGE ISSUES VIA LINEAR FUNCTIONS}

\section{Abstract}

This work aims to show results and data analysis of the last 3 questions of an evaluation, which included a total of 6 questions (the first 3 questions were analyzed in another article). The assessment was proposed in the classroom of a federal public college located in a large city, state capital, for students in a

${ }^{1}$ Doutor em Educação Matemática pela UNIBAN - Universidade Bandeirante de São Paulo (2012). Professor Associado do Departamento de Análise, Instituto de Matemática e Estatística, Universidade Federal Fluminense - UFF. Contato: paulojorge@id.uff.br. 
class of the 1st grade of high school and was delivered by 12 students (there were 10 absences, and 6 students did not attend). delivered). The purpose that guided the study was to describe the nature of the subjects' solutions - according to the level of mobilization of proportional reasoning in relation to the determination of percentage increases and decreases in values and amounts in order to know and identify how the nature of the teaching interventions directed by the teacher. The evaluation took place in the class following the end of the development and exploration of concepts associated with the exercise of proportional reasoning via concepts pertinent to linear functions, during reflections made when solving Financial Mathematics questions, over 4 classes, duration of 40 minutes each. It is a qualitative research, in the form of strategic action-research, according to which the analysis of the data constituted the motto to point out indications regarding the appropriation of proportional reasoning. The results showed that the methodology and the resolution procedures developed for this universe of situations did not attract the study subjects as an alternative of resolution.

Keywords: Proportionality; Percentage; Proportional reasoning; Linear functions.

\section{Introdução}

Este trabalho é recorte de uma pesquisa, em fase inicial, a qual objetiva responder a seguinte questão: "Que situações de aprendizagem um professor de matemática precisa selecionar, dirigir e propor a seus alunos de modo a identificar e conhecer como o raciocínio proporcional é exercitado por eles quando da resolução de problemas que estejam relacionados às temáticas Matemática Financeira e Educação Financeira Crítica, de modo a compreender quais dificuldades eles enfrentam e como proceder para ajudá-los a superar essas dificuldades?"

O estudo objeto deste trabalho tem o propósito de identificar, conhecer e analisar como se dá a apropriação de competências associadas com a resolução de problemas de percentagem por meio do exercício do raciocínio proporcional (RP) presente nos conceitos de funções lineares.

Foram objeto de análise de dados as produções constantes da resolução e respostas fornecidas a um conjunto de 6(seis) problemas, em uma avaliação que foi aplicada na aula final do estudo. Por conta do limitado espaço disponível para a publicação das análises de todos os dados colhidos no estudo, foi feita a repartição em dois trabalhos: o primeiro, publicado em Teixeira (2020b), versou sobre a análise de dados dos 3 primeiros problemas da avaliação, com a menção de a avaliação dos outros 3 problemas ser feita em artigo futuro. Agora, neste trabalho, será feita a análise dos dados sobre os 3 últimos problemas da avaliação, seguida de considerações acerca de todo o estudo.

A intervenção objeto do estudo permitiu que os sujeitos conhecessem uma alternativa para a apropriação do conceito de proporcionalidade direta segundo 
o particular significado de percentagem, por meio do constante exercício do RP. Os momentos de desenvolvimento da temática ao longo do estudo contaram com a proposição, exploração, resolução, reflexão e discussões de um conjunto de 13(treze) diferenciados problemas financeiros (particularmente, acerca de aumentos ou decréscimos percentuais de quantias ou valores), e paralelamente foram desenvolvidos conceitos e procedimentos por meio de funções lineares.

Durante a resolução dos 13 problemas - realizada em momentos que antecederam a avaliação (que tiveram lugar na sala de aula da turma, ao longo de 4 aulas, duração de 40 minutos cada) - os sujeitos do estudo foram instados a desenvolver, exercitar e aplicar o RP por meio do particular significado associado ao conceito de proporcionalidade, como uma alternativa para a resolução e a obtenção da solução para cada um dos 13 problemas. Mas, por conta do espaço disponível nesta publicação, apenas será possível apresentar a análise dos dados de um dos 3 últimos problemas constantes da avaliação. 0 objetivo é mostrar como o estudo foi encaminhado, e quais resultados foram obtidos.

\section{Referencial teórico}

Para a análise das resoluções e respostas aos problemas constantes da avaliação reportamo-nos à Tall e Vinner (1981), os quais definem imagem conceitual como a estrutura cognitiva total construída na mente de uma pessoa, a respeito de determinado conceito matemático, abrangendo todas as ideias, imagens mentais, impressões, representações visuais e descrições verbais relativas às propriedades e processos que envolvem aquele conceito. Segundo Tall e Vinner (1981, p.2)

Como resultado e por meio de experiência de todos os tipos que uma pessoa se vê envolvida ao longo do tempo a imagem de um conceito vai se constituindo e se transformando continuamente quando ela passa pelo enfrentamento de novos estímulos [...].

Também reportamo-nos à perspectiva de Fischbein (1994) segundo aspectos presentes na atividade matemática. O componente intuitivo (ou, simplesmente, compreensão intuitiva, cognição intuitiva, solução intuitiva) "diz respeito a uma compreensão que uma pessoa considera auto evidente", Fischbein (1994, p. 232). Essa compreensão é de tal maneira aceita pela pessoa que ela é capaz de aceitar uma ideia ou um conhecimento sem sequer questionar de que é preciso que haja necessidade de encontrar um tipo de justificativa que venha a legitimá-la.

É indispensável que se ofereça aos alunos um processo educativo que valorize a apropriação do componente formal, considerando que compreender o que seja rigor e coerência em Matemática não é uma tarefa que o aluno adquira de maneira espontânea sem prescindir do professor [...] (FISCHBEIN, 1994, p. 232). 
Segundo o autor o conhecimento acerca dos componentes formais não garante o necessário para o enfrentamento de quaisquer problemas, pois "o domínio de técnicas e procedimentos isento do conhecimento de argumentos que justificam a utilização dessas técnicas pode não ser suficiente para a resolução de problemas que fogem ao padrão", Fischbein (1994, p. 232).

\section{Propósitos e sujeitos do estudo}

O estudo teve início com o propósito de analisar a produção dos alunos no tocante às resoluções e comunicação de respostas a um conjunto de problemas, os quais seriam propostos ao longo de todo o trimestre letivo em curso. Nesse desenrolar os conceitos próprios às funções lineares e o particular significado de proporcionalidade por meio do exercício do RP, também seriam apresentados e explorados para cada um dos 13 problemas, selecionados serem estudados nos momentos de desenvolvimento da temática por meio de questionamentos do professor aos alunos, da seguinte maneira: Como vocês poderiam resolver este problema à luz dos conceitos de proporcionalidade presentes nas funções lineares?

Para atender a esse propósito um planejamento prévio foi elaborado tomando por base o objetivo de desenvolver o conceito de proporcionalidade e o respectivo significado conforme as grandezas e a situação envolvidas em cada problema. Assim, para cada problema, uma vez que a existência de proporcionalidade e as variáveis envolvidas tenham sido identificados e se a proporcionalidade era do tipo direta ou inversa, o conhecimento matemático necessário para encaminhar a resolução deveria ser mobilizado, então. Ou seja, cada problema se configuraria como um disparador para o pertinente ensino aprendizagem dos conceitos e procedimentos associados.

Mas, antes mesmo que o propósito inicial tivesse sido encaminhado como previsto no desenho inicial, o professor encaminhou o estudo no sentido de identificar e conhecer como os sujeitos mobilizariam os conhecimentos acerca do exercício do RP na resolução de problemas relacionados com aumentos ou decréscimos percentuais. E por conta da permissividade da metodologia, assim foi feito. Então, um novo propósito de trabalho foi elaborado e proposto ser desenvolvido com a turma. e o que foi previsto foi cumprido.

Uma vez feita a seleção dos 13 problemas que seriam objeto do estudo (que versaram sobre aumentos ou decréscimos percentuais), o trabalho foi desenvolvido por meio de reflexões e discussões acerca da mobilização de conhecimentos e competências que dessem conta de resolvê-los. 0 desenvolvimento da investigação incluiu os diálogos havidos entre os sujeitos do estudo em grupos menores de 4 alunos, e entre eles e o pesquisador, no lócus do estudo e da avaliação: a sala de aula, ao longo de 4(quatro) aulas, com duração de 40 (quarenta) minutos cada. Ao final desse momento, e sem aviso prévio, uma ficha de avaliação contendo 6(seis) problemas envolvendo a temática foi proposta aos sujeitos do estudo, na aula seguinte ao término do estudo, sem aviso prévio, e com igual duração de uma aula, para que cada um a resolvesse, e fizesse a sua entrega ao final. Os 6 problemas selecionados para (c)(1) 
a avaliação, embora distintos dos 13 problemas que foram trabalhados em sala de aula, guardaram relativa similaridade entre si.

Considerou-se como sujeitos do estudo objeto deste trabalho os 12 alunos de uma turma do $1^{0}$ Ano do Ensino Médio presentes na sala de aula onde a avaliação foi proposta: um colégio público pertencente à Rede Federal de Ensino Básico, localizado em grande cidade, capital do estado, na qual o autor foi o professor de Matemática, no segundo semestre do ano letivo de 2019. Neste dia de avaliação um total de 10 alunos não foram a aula. e 6 alunos que estavam presentes e fizeram a avaliação (em parte ou na totalidade) não quiseram entregar a ficha de avaliação, ao final.

\section{Resultados e discussões}

No estudo foi adotada a metodologia "Design Experiment in Educational Research", de Cobb; Confrey; DiSessa; Lehrer e Schauble (2003). A escolha da metodologia foi feita em função de ela ser dotada de flexibilidade de adaptação ao desenho inicial proposto considerando as produções fornecidas pelos sujeitos do estudo. Segundo essa metodologia, preliminarmente é elaborado um desenho básico flexível, que pode ou não sofrer modificações ao longo de todo o processo do estudo. Por conta de possíveis modificações, a metodologia permite que sejam geradas novas conjecturas, como é preciso, as quais precisam ser testadas a posteriori. Além do mais tal metodologia prevê a elaboração de experimentos de ensino de conteúdos da Matemática, com vistas à obtenção de inovações. Salienta-se que o professor-pesquisador responsabilizou-se por identificar as adaptações que se fizeram necessárias implementar, ao longo do estudo, ao assumir o papel de orientador, intervindo somente em momentos críticos considerados por ele como de bloqueio, durante o desenrolar das tarefas propostas.

A análise dos dados levou em conta a investigação das trajetórias dos sujeitos do estudo segundo a identificação das dificuldades e os possíveis avanços alcançados. Com base na metodologia escolhida o desenrolar do estudo seguiu, segundo um processo que se configurou como iterativo, cíclico e flexível. Conforme apontam os autores da metodologia há algumas formas diferenciadas de sua aplicação, mas no estudo em questão ela foi aplicada a um pequeno grupo de sujeitos com o propósito de analisar as suas trajetórias, de maneira mais amiúde.

O estudo foi desenvolvido em um ambiente natural (sala de aula) para a coleta direta dos dados, onde o pesquisador exerceu papéis que o colocaram como o instrumento central para tal: professor e pesquisador. Nesse ambiente o pesquisador não se valeu do propósito de recriar experimentalmente problemas de estudo que não os que foram propostos no início do trabalho investigativo, e sobre os quais se propôs a estudar, observar e coletar dados para o estudo. O pesquisador procurou estar atento a todos os elementos presentes nas situações estudadas, sabendo de antemão que aspectos por vezes considerados triviais e aparentemente considerados sem importância podem se transformar em informações valiosas, para compor o conjunto de dados do 
estudo. Ou seja, aspectos carregados de significados, os quais ajudam na compreensão dos fenômenos em questão. Considerando os aspectos básicos que caracterizam uma investigação qualitativa, como os presentes em Franco (2005), considerou-se que o estudo se caracterizou como uma investigação de natureza qualitativa.

\section{Conhecimento do conteúdo grandezas proporcionais}

No tocante ao conteúdo matemático grandezas proporcionais, que será explorado nesta seção, reportamo-nos às considerações feitas por Lima (1991).

Se diz que "a grandeza y é diretamente proporcional à grandeza $x$ " quando as 2(duas) condições especificadas a seguir são satisfeitas: (i) y (ou f) é uma função crescente de $x$; (ii) se multiplicarmos $x$ por um número racional positivo $\mathrm{m}$ o valor correspondente de y também fica multiplicado por $\mathrm{m}$. Em linguagem matemática, escreve-se assim: $f(m . x)=m . f(x)$, para todo valor de $x$ e todo número racional positivo $\mathrm{m}$. Por conta dessa condição, existe um número $\mathrm{k}$, chamado de "constante de proporcionalidade" entre $x$ e $y$, tal que $f(x)=k . x$, para todo valor de $x$. Mas, atenção: na definição de grandezas diretamente proporcionais será preciso que as duas condições acima sejam atendidas. Uma só condição não garante proporcionalidade!

Ressalte-se que a função $f$, como acima, é dita uma função linear, pois além da propriedade (ii) ser atendida também a propriedade aditiva $f(a+b)=$ $k \cdot(a+b)=k \cdot a+k \cdot b=f(a)+f(b)$ é satisfeita, para quaisquer valores das grandezas a e b. Assim, podemos estabelecer a seguinte relação: "a grandeza y é diretamente proporcional à grandeza $x$ " se e somente se a função $f(x)=y$ é função linear. Portanto, o modelo matemático associado às funções lineares é um modelo que atende satisfatoriamente à relação entre valores ou quantias e percentuais, no seguinte sentido: variações percentuais estão em consonância com variações de valores ou quantias, e vice versa. O modelo matemático funções lineares é um modelo que atende às condições de proporcionalidade entre 2(duas) grandezas $x$ e $y$. Considere, pois, que $x 1, \times 2$ e $x 3$ são valores distintos assumidos pela grandeza $x$ e, correspondentemente, y1, y2 e y3 são valores distintos assumidos pela grandeza $y$, como mostrado na Figura 1, a seguir.

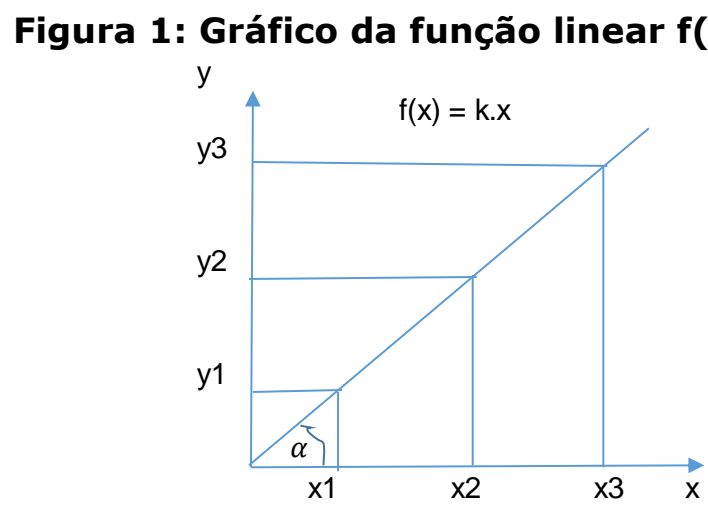

Fonte: Dados do estudo 
De modo que y seja diretamente proporcional a $x$ (proporcionalidade direta), é necessário e suficiente que $y 1=f(x 1)=k . x 1, y 2=f(x 2)=k . x 2$ e $\mathrm{y} 3=\mathrm{f}(\mathrm{x} 3)=\mathrm{k} . \mathrm{x} 3$. Tais igualdades implicam que $\mathrm{k}=\frac{y_{1}}{x_{1}}=\frac{y_{2}}{x_{2}}=\frac{y_{3}}{x_{3}}=\ldots$ O gráfico da Figura 1, acima, mostra-nos que $\operatorname{tg} \alpha=\mathrm{k}$.

Assim, tal qual Lima (1991, p.131) defende, se diz que 2(duas) grandezas y e x são diretamente proporcionais (que a proporcionalidade entre elas é direta) quando entre elas há uma correspondência, de tal modo que ao se multiplicar a grandeza $\mathrm{x}$ por um número racional positivo a quantidade correspondente de $\mathrm{y}$ também fica multiplicada por esse mesmo número.

O RP, exercitado em problemas com proporções, por exemplo, requer do sujeito que está encaminhando a resolução do problema um olhar diferenciado por conta de a abordagem exigir anterior avaliação, sob vários pontos de vista. Mormente para identificar situações em que o que está em questão é a presença da não-proporcionalidade entre 2(duas) grandezas. A grandeza "área de um círculo", por exemplo, não é diretamente proporcional ao aumento ou diminuição da grandeza "medida do raio do mesmo círculo". Sobre essa questão, é preciso cuidado ao ensinar, conforme aponta Teixeira (2020a). Uma das aplicações da noção de proporcionalidade é a conhecida "Regra de Três". Na "Regra de Três" se tem uma grandeza y (direta ou inversamente) proporcional a $\mathrm{x}$, isto é: $\mathrm{y} 1=$ $f(x 1)$, y2 $=f(x 2)$. O problema consiste em encontrar um dos 4(quatro) valores $x 1, x 2, y 1$ ou $y 2$, conhecendo-se os outros 3 valores. A partir daí conclui-se, portanto, que "Se y é diretamente proporcional a $x$, então a "Regra de Três" é dita direta" e "Se y é inversamente proporcional a $x$, então a "Regra de Três" é dita inversa".

Ressalte-se que primeiramente será preciso garantir ser y diretamente proporcional a x para que a "Regra de Três" seja resolvida, pois a partir daí é que se tem: $y 1=f(x 1)=k \cdot x 1 \rightarrow k=\frac{y 1}{x 1}(1)$ e $y 2=f(x 2)=k \cdot x 2 \rightarrow k=\frac{y 2}{x 2}$ (2). De (1) e (2): $\frac{\mathrm{y} 1}{\mathrm{x} 1}=\frac{\mathrm{y} 2}{\mathrm{x} 2} \rightarrow \mathrm{y} 2=\frac{\mathrm{y} 1 \cdot \mathrm{x} 2}{\mathrm{x} 1}$ ou $\mathrm{y} 1=\frac{\mathrm{y} 2 \cdot \mathrm{x} 1}{\mathrm{x} 2}$ ou $\mathrm{x} 2=\frac{\mathrm{y} 2 \cdot \mathrm{x} 1}{\mathrm{y} 1}$ ou $\mathrm{x} 1=$ $\frac{\mathrm{y} 1 . \mathrm{x} 2}{\mathrm{y} 2}$.

\section{Descrição de resolução de um dos 13 problemas constante do estudo}

Como dito, um total de 13(treze) problemas foram propostos, refletidos, discutidos e resolvidos pelos sujeitos do estudo em sala de aula, com a mediação do professor, em processo dialógico. Em prosseguimento, e por conta de espaço disponível, vamos apresentar como se deu a discussão, reflexões e discussões acerca da resolução e solução de apenas um problema: o Problema 2, cujo propósito é o de calcular o novo valor de uma grandeza após a concessão de um desconto percentual sobre o valor original. Ressalte-se que no artigo de

Teixeira (2020b), o Problema 1 foi igualmente apresentado. Eis o enunciado do Problema 2, como a seguir:

a) Um par de sapatos custa $\mathrm{R} \$ 80,00$ caso a compra seja feita com o uso do cartão de crédito, mas se o pagamento é feito com o uso de dinheiro é 
concedido um desconto de 5\%. Qual o valor a pagar pelo par de sapatos com o uso de dinheiro? b) Se o mesmo par de sapatos tiver o seu preço alterado: passar a custar $R \$ 60,00$ ao invés dos atuais $R \$ 80,00$, qual percentual de desconto foi concedido?

Na Figura 2, a seguir, o gráfico à esquerda mostra-nos que as variáveis y: percentual e x: preço (valor ou quantidade) atual estão em proporcionalidade direta. Por conta disso, o gráfico à direita mostra-nos que também as variáveis $\mathrm{x}$ : preço atual e y: percentual estão em proporcionalidade direta, segundo 0 modelo matemático função linear, que será adotado aqui.

Figura 2 - Acréscimos ou decréscimos percentuais via aplicação de uma função linear
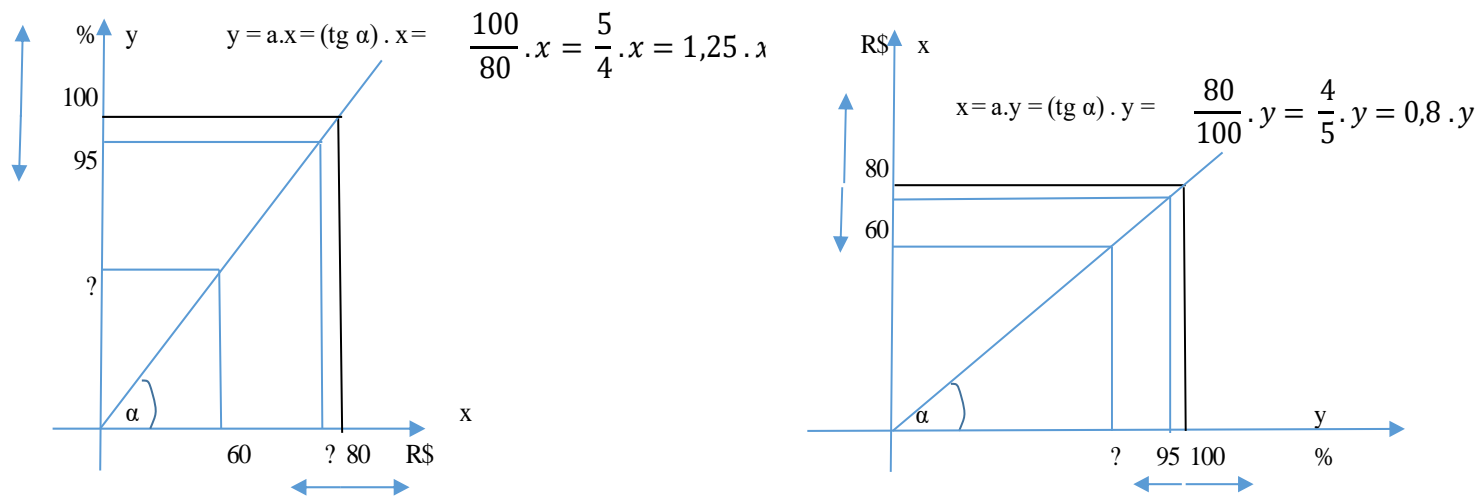

Fonte: Dados do estudo.

Nos dois gráficos, o percentual de $100 \%$ está em consonância com o valor $\mathrm{R} \$ 80,00$. O gráfico à esquerda, na Figura 2, acima, mostra-nos o valor percentual $y=100 \%$ como imagem do valor monetário $x=R \$ 80,00$ por meio da função linear $f: A \rightarrow B$ ( $A$ o conjunto de valores em reais positivos, e $B$ o conjunto de percentuais positivos), segundo o modelo matemático função linear. Então: $\mathrm{f}(\mathrm{x})=\mathrm{y}=\mathrm{a} \cdot \mathrm{x}=(\operatorname{tg} \mathrm{a}) \cdot \mathrm{x}=\frac{100}{80} \cdot \mathrm{x} \rightarrow \mathrm{f}(\mathrm{x})=\frac{5}{4} \cdot \mathrm{x}=1,25 \cdot \mathrm{x} \rightarrow \mathrm{y}=1,25 \cdot \mathrm{x}$.

O percentual correspondente ao desconto de 5\%, para a compra feita em dinheiro, é de $100 \%-5 \%=95 \%$ do valor original. Assim, y $=95 \%$ é imagem de certo valor $x$ pela função $f$, valor esse que precisa ser determinado. Logo, $\mathrm{f}(\mathrm{x})=\mathrm{y}=\frac{5}{4} \cdot x=95 \% \rightarrow \mathrm{x}=\frac{4.95}{5}=\mathrm{R} \$ 76,00$. Nesse caso se diz que o valor $\mathrm{R} \$ 76,00$ corresponde a $95 \%$ do valor original $\mathrm{R} \$ 80,00$. O gráfico da função linear $\mathrm{f}: \mathrm{A} \rightarrow \mathrm{B}$ assegura esse resultado, uma vez que tg $\mathrm{a}=\frac{95}{76}=\frac{5}{4}=1,25$. É importante compreender que a manutenção de proporcionalidade direta é uma garantia assegurada pelo modelo matemático função linear, como foi mostrado acima. Assim, $f(80)=100 \%$, assim como $f(76)=95 \%$. Na Figura 2, acima, o gráfico à direita mostra-nos que as variáveis $x$ : preço (valor ou quantidade) atual e $y$ : percentual estão em proporcionalidade direta segundo o modelo matemático função linear. Assim, o percentual de 100\% está em consonância com o valor $\mathrm{R} \$ 80,00$. O gráfico mostra-nos o valor monetário $\mathrm{x}=\mathrm{R} \$ 80,00$ como imagem do valor percentual $y=100 \%$ por meio da função linear $f: B \rightarrow A$ ( $B$ o conjunto de percentuais positivos e $A$ o conjunto de valores em reais positivos), segundo 0 (c) (1) ( 
modelo matemático linear. Então: $\mathrm{f}(\mathrm{y})=\mathrm{x}=\mathrm{a} \cdot \mathrm{y}=(\operatorname{tg} \mathrm{a}) \cdot \mathrm{y}=\frac{4}{5} \cdot y=0,8 \cdot \mathrm{y} \rightarrow \mathrm{x}=$ $0,8 \cdot y$.

O percentual correspondente ao desconto de $5 \%$ para a compra feita em dinheiro é de $100 \%-5 \%=95 \%$ do valor original. Assim, o valor monetário $x$, que precisa ser determinado, é imagem de $y=95 \%$ pela função $f$. Portanto: $x$ $=0,8 \cdot y=0,8 \times 95=R \$ 76,00$. O gráfico da função linear $f: B \rightarrow A$ assegura esse resultado, uma vez que $\operatorname{tg} a=\frac{76}{95}=\frac{4}{5}=0,8$.

O conceito matemático envolvido na resolução pode ser compreendido assim: "Multiplicar certa quantia (valor) por um número positivo inferior a $1=$ $100 \%$ aponta para um desconto concedido sobre o valor original, isto é, um desconto sobre a quantia(valor)" original. Ou, de modo similar: "multiplicar certa quantia (valor) por um número superior a $1=100 \%$ aponta para um aumento do valor original, isto é, uma aumento da quantia(valor)". Se diz que o conceito proporcionalidade direta foi apropriado por um sujeito - para o particular significado de aumentos ou decréscimos percentuais, segundo o modelo matemático função linear - quando o conhecimento necessário para a resolução de problemas monetários foi por ele compreendidos e mobilizados em diferentes contextos, a exemplo dos que foram objeto de discussão por ocasião do Problema 2, como acima.

Um importante conhecimento diz respeito à compreensão de que o produto de certo valor inicial positivo por 0,75 aponta para um decréscimo (desconto) percentual de $1-0,75=0,25=25 \%$, que é concedido sobre o valor inicial. Na verdade, multiplicar qualquer valor positivo por um certo valor positivo menor que 1 aponta para o resultado ser um valor menor que o inicial. Importante também compreender que multiplicar certo valor inicial por 0,75 é equivalente a multiplicar o valor inicial por 3 , e em seguida dividir o resultado obtido por 4 (ou então, dividir o valor inicial por 20 e em seguida multiplicar o resultado obtido por 15). Ou seja, compreender que dividir por 4 e em seguida multiplicar por 3 é equivalente a dividir por 20 e em seguida multiplicar por 15, em clara referência ao conceito de frações equivalentes. Também, compreender que o produto de certo valor inicial positivo por 1,45, por exemplo, aponta para um acréscimo (aumento) percentual de 1,45 - $1=0,45=45 \%$, que é aplicado sobre o valor inicial. Na verdade, multiplicar qualquer valor positivo por um certo valor positivo maior que 1 aponta para o resultado ser um valor maior que o inicial. Igualmente, compreender acerca da divisão por um valor menor que 1 ou por um valor maior que 1 . Estas considerações precisam fazer parte de um conjunto de conhecimentos matemáticos básicos tal qual o domínio que se espera da tabuada, mesmo que se considere elas serem simples ser asseguradas após cálculos feitos com o uso de uma calculadora. E na ausência da calculadora? Mas, não podem se configurar como algo que se aprende e se usa, desprovido de entendimento acerca das razões do porquê tal acontece. Acerca dessa questão, Fischbein (1994, p.232) chama-nos atenção no sentido de que "o domínio de técnicas e procedimentos isento do conhecimento de argumentos que justificam a utilização dessas técnicas pode não ser suficiente para a resolução de problemas que fogem ao padrão". Pouco adianta usar a calculadora 
para fazer o produto $35 \times 1,2$, por exemplo, observar o valor 42 no visor, e não saber concluir a respeito do conceito que está envolvido? O que representa, percentualmente, o valor 42 em relação ao valor 35 ?

As diferentes representações: frações, números decimais, percentuais e as competências necessárias para encaminhar a resolução de um problema (financeiro ou não) são importantes, e precisam ser compreendidas e apropriadas pelo aluno nas situações em que ele dispõe ou não de uma calculadora. O uso da calculadora não dispensa a compreensão de conceitos como os apresentados acima, ou seja, a calculadora não garante a obtenção da solução em certos casos, para certos tipos de problemas, pois embora o seu uso permita determinar diretamente o valor de 95\% de 80,00 teclando 80,00 x 95\% = 76 (essa representação não é apresentada na forma de unidades monetárias), por exemplo, para determinar o valor percentual de $60 \mathrm{em} \mathrm{80:} \frac{60}{80}=0,75 \mathrm{de}$ modo indireto será preciso interpretar o valor 0,75 segundo o significado percentual correspondente a $75 \%$, e concluir que 60 é corresponde ao decréscimo de $25 \%$ sobre 80 .

\section{Análise dos dados}

As resoluções e as respostas para os problemas constantes da Ficha de Avaliação fazem parte de um conjunto de dados que deu origem à análise e considerações objeto deste trabalho. Para a análise dos dados que foram produzidos pelos sujeitos do estudo quando da resolução e resposta para cada problema proposto na avaliação, os diferentes tipos de resoluções foram categorizadas por meio da descrição da natureza das soluções segundo níveis de compreensão do conceito de proporcionalidade direta e o exercício do RP ou não.

Assim, com o objetivo de estabelecer os níveis de compreensão do conceito de proporcionalidade direta e o exercício do RP presente ou não nas resoluções dos problemas propostas na avaliação o autor estabeleceu 7(sete) níveis de resolução/solução, conforme a seguir:

Nível 0: Resolução e resposta em branco;

Nível 1: Resposta obtida por meio de cálculos, sem indício do exercício do RP;

Nível 2: Resposta obtida por meio de cálculos, com indício de proporcionalidade via aplicação da Regra de Três;

Nível 3: Primeiras aproximações à solução por meio do exercício do RP;

Nível 4: Resposta obtida por meio de alguma noção do exercício do RP;

Nível 5: Resolução obtida via exercício do RP, em parte, acompanhada de cálculo(s) (multiplicação ou adição ou subtração) para a obtenção da resposta;

Nível 6: O RP foi exercitado, integralmente, até a obtenção da resposta final.

As frequências de respostas (corretas e incorretas) para todos os problemas da avaliação, apresentadas pelos 12 sujeitos do estudo, foram distribuídas por nível de compreensão e serão apresentadas, a seguir, em uma tabela. Os resultados produzidos com a experiência e a análise de dados (que (c) (1) 
serão mostrados em seguida) constantes das resoluções que foram apresentadas pelos sujeitos do estudo, foram determinantes para as reflexões e considerações acerca do papel e relevância do estudo. Em prosseguimento, apresentamos os enunciados dos 6 (seis) problemas constantes da Ficha de Avaliação:

Problema 1: Se a quantidade de produtos à venda em uma loja cresceu de 40 para 240, de quanto foi o porcentual de aumento na quantidade de produtos?

Problema 2: Se um par de sapatos custa $\mathrm{R} \$ 30,00$ caso a compra seja feita via cartão de crédito, e é concedido um desconto de $8 \%$ se o pagamento for feito à dinheiro, qual o valor a pagar com o uso de dinheiro?

Problema 3: Se uma bermuda custa $\mathrm{R} \$ 40,00$ caso a compra seja feita via cartão de crédito e, se o pagamento for feito à dinheiro, o preço passa a ser $\mathrm{R} \$ 34,00$, qual percentual de desconto é concedido quando a bermuda é paga em dinheiro?

Problema 4: Se uma bermuda custa $\mathrm{R} \$ 40,00$, hoje, e para o próximo mês haverá um acréscimo de $11 \%$ sobre este valor, qual será o novo valor a pagar por esta mesma bermuda no próximo mês?

Problema 5: Se um par de tênis custa $\mathrm{R} \$ 50,00$, hoje, e para o próximo mês ele vai custar $R \$ 56,00$, qual será o percentual de aumento sobre o preço de hoje do par de tênis?

Problema 6: Dividir o valor original $\mathrm{R} \$ 30,00$ de um produto por 1,25 aponta para um aumento ou para um decréscimo? Em termos percentuais, de quanto é esse aumento ou esse decréscimo?

O Quadro 1, a seguir, mostra a frequência absoluta de problemas deixados em branco; de respostas corretas (C) e o quantitativo destas de Nível 2; e a frequência absoluta de respostas erradas (E) e os quantitativos destas de Nível 1, Nível 2 e Nível 4, isto é, quantitativos de respostas por níveis de incompreensão do exercício do Raciocínio Proporcional. Como deveria ser, foram considerados todos os 12 sujeitos do estudo, e a totalidade dos 6 problemas constantes da Ficha de Avaliação.

Quadro 1: Quantitativos de respostas para os problemas presentes na avaliação: corretas(C) e erradas(E) (por nível de incompreensão do Raciocínio Proporcional).

\begin{tabular}{|c|c|c|c|c|c|}
\hline $\begin{array}{c}\text { Quantidade } \\
\text { Problema }\end{array}$ & $\begin{array}{c}\text { No de respostas } \\
\text { em branco }\end{array}$ & $\begin{array}{c}\text { No de respostas } \\
\text { corretas }\end{array}$ & $\begin{array}{c}\text { No de respostas corretas } \\
\text { de Nível 2 }\end{array}$ & $\begin{array}{c}\text { No de respostas } \\
\text { erradas }\end{array}$ & $\begin{array}{c}\text { No de respostas } \\
\text { erradas por Nível }\end{array}$ \\
\hline Problema 1 & 0 & 10 & 7 & 2 & N2 e N4 \\
\hline Problema 2 & 1 & 9 & 6 & 2 & N1 e N2 \\
\hline Problema 3 & 1 & 9 & 6 & 2 & N1 e N2 \\
\hline Problema 4 & 1 & 9 & 4 & 2 & N2 \\
\hline Problema 5 & 0 & 10 & 5 & 2 & N1 e N2 \\
\hline Problema 6 & 0 & 10 & 5 & & N1 \\
\hline
\end{tabular}

Fonte: Dados do estudo. 
As análises dos dados e considerações pertinentes, relativos às resoluções e soluções para os 3 primeiros problemas, estão apresentados em XxXXXXXX $(x x x b)$.

Em prosseguimento, é apresentada a análise dos dados produzidos com as resoluções e soluções para os 3 últimos problemas propostos na Ficha de Avaliação. As Figuras 3 e 4, a seguir, mostram resoluções corretas, ambas solução Nível 6, que foram apresentadas pelo aluno A9 para os problemas 4 e 5 , respectivamente.

\section{Figura 3 - Resolução correta, apresentada pelo aluno A9 para o Problema 4. Resolução Nível 6.}

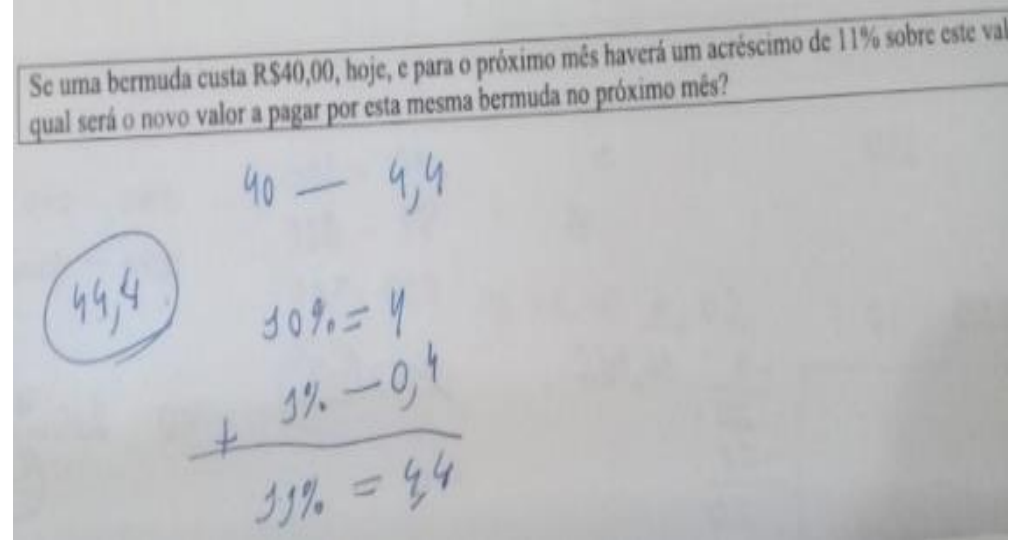

Fonte: Dados do estudo

O aluno A9 considera as grandezas preço e porcentagem como diretamente proporcionais e na resolução apresentada exercita o RP de maneira correta até a obtenção da resposta final, solução Nível 6 , ao determinar $10 \%$ de 40; depois $1 \%$ de 40 ; e a indicação de duas somas com resultados: $11 \%$ e 4,4. De imediato, o resultado da soma de $40 \mathrm{com} \mathrm{4,4,} \mathrm{e} \mathrm{a} \mathrm{resposta} \mathrm{44,4.} \mathrm{Chama} \mathrm{a}$ atenção o fato de A9 não identificar os valores como dinheiro (unidades monetárias do real). Sequer se refere aos 40 centavos. Poderia ter escrito a proporcionalidade direta $\mathrm{R} \$ 44,40-111 \%$, mas preferiu fazer a comunicação da resposta diretamente, com o valor 44,4 .

O problema 4 apresentou 6 resoluções Nível 2, com 2 delas incorretas. Evidencia o aspecto algorítmico do cálculo direto de $11 \%$ de $\mathrm{R} \$ 40,00$ e a posterior soma de $\mathrm{R} \$ 40,00$ com este valor parece transparecer tratar-se de uma prática costumeira, aprendida nos anos anteriores. Possivelmente, tal prática foi favorecida pela presença de valores inteiros do percentual e do preço da bermuda. Fato é que, independentemente do Nível de resolução, foi um problema em que 9 dos 12 sujeitos acertaram a resposta, 2 erraram e um único a deixou em branco. 


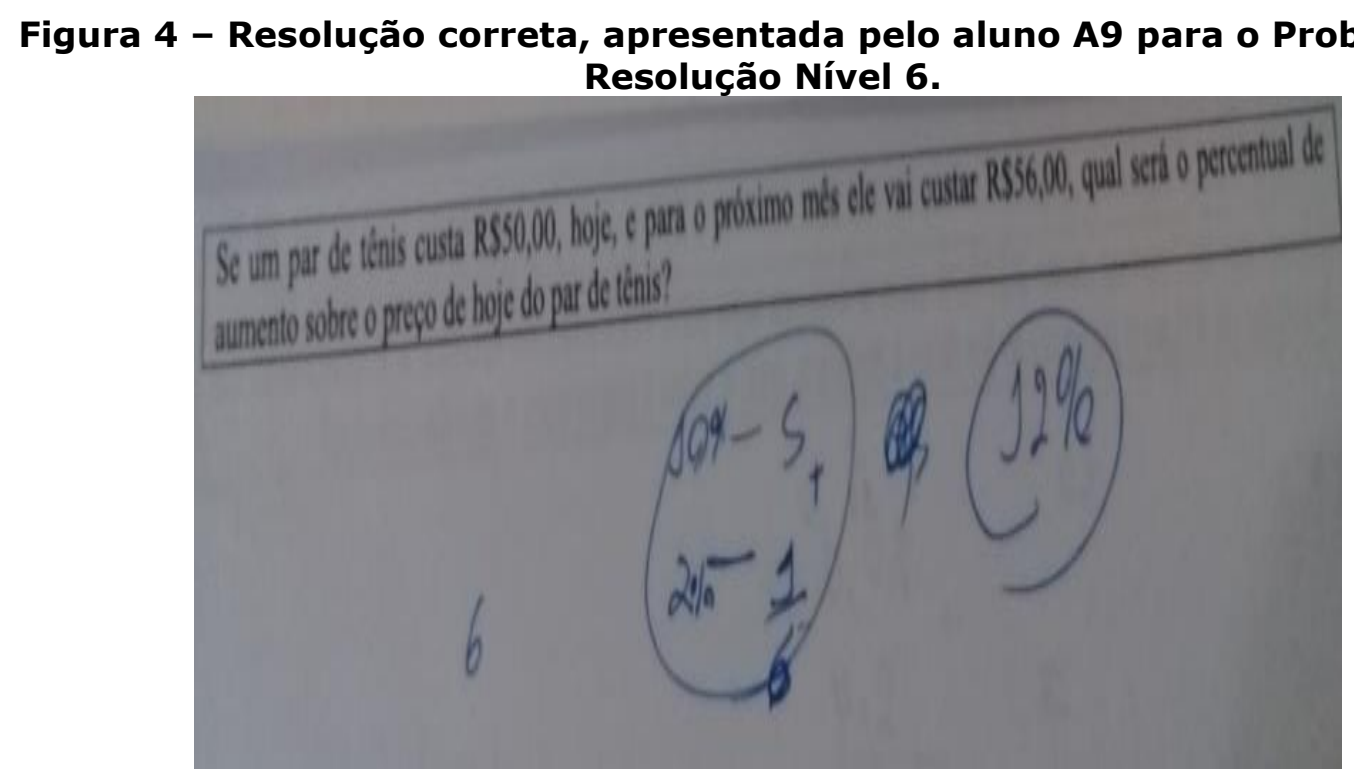

Fonte: Dados do estudo.

Para o problema 5 o aluno A9 considera as grandezas preço e porcentagem como diretamente proporcionais e, na resolução apresentada na Figura 4, acima, exercita o RP de modo correto até a obtenção da resposta final (solução Nível 6 ), assim; determina $10 \%$ de $R \$ 50$; depois $2 \%$ de $R \$ 50$; a indicação da soma $5+1=6$ e, de imediato, o resultado da soma de $10 \%$ com $2 \%$. O Problema 5 apresentou bom quantitativo de soluções Nível 2: 6 em 12, com uma incorreta, e soluções Nível 1: 4 em 12, sendo uma incorreta. Possivelmente houve certo favorecimento, por conta dos valores inteiros: $R \$ 50,00$ e $R \$ 56,00$ e a proximidade entre eles. A Figura 5, a seguir, mostra a resolução do aluno A5, para o Problema 6.

Figura 5 - Resolução correta, apresentada pelo aluno A5 para o Problema 6. Resolução Nível 2.

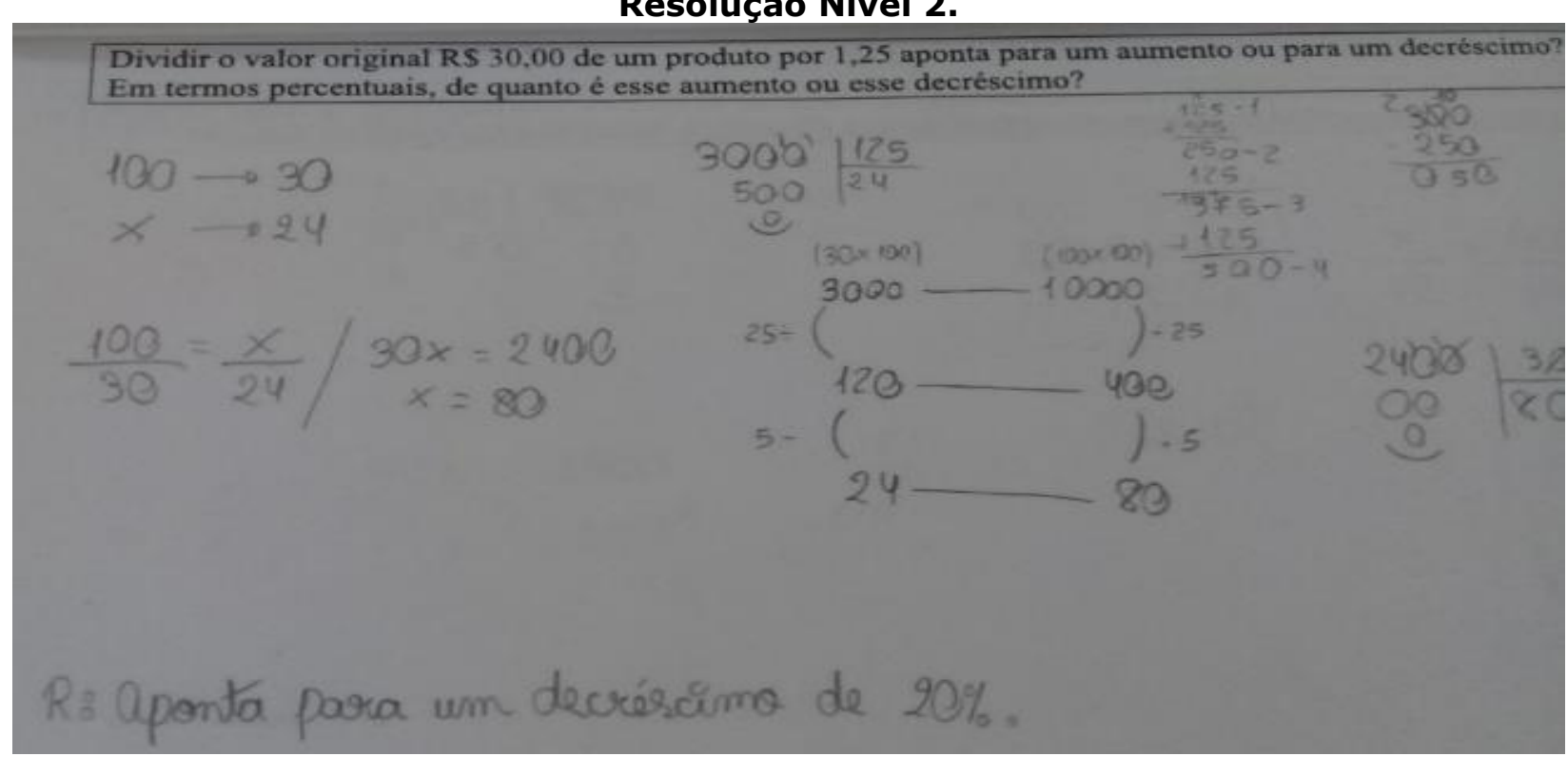

Fonte: Dados do estudo. 
De início, com o uso de uma Regra de Três, para obter $x=80$. Observase que as formas apresentadas ao lado da referida Regra de Três foram consideradas por A9 como confirmatórias ao resultado obtido, segundo nosso entendimento. Ou seja, uma garantia de que a sua resolução e resposta estavam corretas. Tal ação só se materializou após a resolução ter sido feita em 3 etapas: primeiro, após ter feito a divisão de 30 por 1,25 e encontrado o valor de 24; depois, uma vez tendo encontrado o valor 24, estabeleceu uma "Regra de Três" com esse valor, o valor inicial e o percentual de $100 \%$, obtendo o valor percentual de $80 \%$ (de 30) como equivalente ao valor 24 , o que the garantia saber que o percentual original de $100 \%$ passou para $80 \%$ para, só então considerar que o decréscimo foi da ordem de $20 \%$, e assim, comunicar o resultado.

Nos parece que o A5 sente necessidade de confirmar o resultado obtido, por meio da mobilização do RP, ao identificar a divisão de 30 por 1,25 como equivalente ao produto de 30 por 100 e a divisão do resultado por 125 . Também confirma que a divisão por 125 equivale a dividir por 25 , a cada resultado obtido, por 3 vezes em sequência. Sendo assim, estabelece uma "Regra de Três": 30 110. Em prosseguimento, e em equivalência, 3000 - 10000; em seguida, 120 400; e finalmente $24-80$, acabando por confirmar o resultado que obteve por meio da "Regra de Três". Por todas essas razões, consideramos que a resolução apresentada não envolveu a mobilização do RP mas apenas a necessidade de confirmar o resultado obtido de início, razão porque consideramos identificar a solução como de Nível 2.

O problema 6 apresentou apenas 2 respostas incorretas. Nenhum dos 12 sujeitos do estudo deixou o espaço de resolução em branco, e talvez isto se deva ao fato de o sujeito que o vai resolver se sentir confortável em mobilizar o algoritmo da divisão de imediato, devido à facilidade para encontrar o resultado, conforme o texto do enunciado. Nesses casos, o procedimento de resolução apresentado evidencia apenas o aspecto algorítmico na resolução e, portanto, não segue pelo exercício do RP para encaminhá-la. Apenas 2 sujeitos do estudo: alunos A7 e A9, procuraram modelizar a situação por meio da aplicação do conceito de proporcionalidade. Para os demais sujeitos do estudo talvez não tenha sido o caso de considerá-lo como um problema com este viés mas como um problema de divisão.

Ademais, refletimos que o tempo dispendido no estudo talvez não tenha sido suficiente para que os sujeitos do estudo amadurecessem os conceitos relacionados com a apropriação e o exercício do RP. Também, que a seleção dos problemas constantes da Ficha de Avaliação foi feita de tal modo que permitiu à maioria dos sujeitos do estudo resolvê-los por meio de técnicas anteriores como, por exemplo, a Regra de Três, e não pelo exercício do RP.

Os dados constantes do Quadro 1, acima, confirmam esta constatação ao mostrar-nos quão grande foi a incidência de resoluções Nível 2 apresentadas pelos sujeitos do estudo para parcela grade dos problemas via o uso algorítmico da Regra de Três (Regra esta, conhecida pelos sujeitos de estudo de experiências anteriores), sem que estes tenham sentido necessidade de 
estabelecer relações de sua utilidade com a temática que foi desenvolvida no estudo. Ou seja, não apresentaram resoluções alternativas via o modelo matemático das funções lineares e, ao fazerem uso da Regra de Três não a relacionaram com o estudo das funções lineares.

Os problemas 4 e 6 foram os que apresentaram menor incidência de soluções Nível 2. O problema 6 não apresentou resposta errada em Nível 2, mas em Nível 1: cálculos. Quando um sujeito do estudo tomou a iniciativa de fazer uso da Regra de Três como uma ação natural, para ele a iniciativa se configura de tal maneira que essa decisão e compreensão é aceita tal qual ele é capaz de aceitar uma ideia ou um conhecimento, sem sequer questionar acerca de que talvez fosse preciso haver necessidade de encontrar uma justificativa que legitime proceder assim. Salientamos essa questão por conta de a resolução que está sendo apresentada pelo sujeito estar fora do escopo de estudo que foi desenvolvido, ou seja, não seguir o propósito para o qual o estudo foi desenvolvido, qual seja a sugestão de experimentar uma resolução diferenciada consoante o estudo feito.

Quanto aos resultados relacionados com as resoluções para os 3 últimos problemas, como mostrados no Quadro 1, acima, reportamo-nos à perspectiva de Fischbein (1994, p. 232), segundo aspectos presentes na atividade matemática, para ressaltar o que o citado autor salienta em relação ao componente intuitivo (ou, simplesmente, compreensão intuitiva, cognição intuitiva, solução intuitiva), no sentido de que tal componente "diz respeito a uma compreensão que uma pessoa considera auto evidente". Segundo Fischbein (1994, p. 232), a compreensão intuitiva é de tal maneira aceita pela pessoa que ela é capaz de aceitar uma ideia ou um conhecimento sem sequer questionar de que é preciso que haja necessidade de encontrar um tipo de justificativa que venha a legitimá-la.

Por outro lado, Fischbein (1994, p232) argumenta que o conhecimento acerca dos componentes formais não garante o necessário para o enfrentamento de quaisquer problemas, pois "o domínio de técnicas e procedimentos isento do conhecimento de argumentos que justificam a utilização dessas técnicas pode não ser suficiente para a resolução de problemas que fogem ao padrão" (Fischbein, 1994, p. 232). Assim, Fischbein (1994) argumenta que

[...] é indispensável que se ofereça aos alunos um processo educativo que valorize a apropriação do componente formal, considerando que compreender o que seja rigor e coerência em Matemática não é uma tarefa que o aluno adquira de maneira espontânea sem prescindir do professor (FISCHBEIN, 1994, p. 232).

Para os problemas constantes da Ficha de Avaliação e o universo dos sujeitos do estudo os resultados mostraram-nos que situação contrária ocorreu neste estudo em relação ao que Fischbein (1994) preconizou, conforme a citação acima. Ou seja, por meio do domínio da conhecida técnica "Regra de Três" os sujeitos de estudo conseguiram resolver grande parte dos problemas tendo, 
inclusive, obtido resultados quantitativos satisfatórios. Talvez isso se deva ao fato de os sujeitos do estudo considerarem que tal técnica seja mais fácil de aplicar que a modelagem via uma função linear ou por conta de se sentiram mais seguros para usá-la que o exercício do RP, considerando que os problemas em questão favoreceram muito tal escolha. Por outro lado, os sujeitos do estudo mostraram desconhecer qualitativamente as razões do porquê tal técnica funciona tão bem para esta categoria de problemas presentes na Ficha de Avaliação, ao fazerem uso indiscriminado da Regra de Três.

Por conta de reflexões feitas à luz das ideias presentes em Fischbein (1994, p.232), o pesquisador-professor considera que talvez os resultados tenham ocorrido por conta da escolha dos problemas que compõem a Ficha de Avaliação. Ou seja, dever-se-ia ter escolhido problemas que não poderiam ser facilmente resolvidos por meio da aplicação direta da Regra de Três e, sim, problemas cuja resolução exigiriam a necessidade de ter de exercitar o RP - de preferência, exercitá-lo via o uso de modelos de funções lineares. Daí a importância que se deve conferir à adequada seleção de problemas que devem compor uma Ficha de Avaliação em estreita conexão com o propósito a que ela se destina. O que nos parece, agora, nesta etapa de avaliação dos dados coletados é que tal preocupação talvez não tenha ocorrido neste estudo, o que lamentamos.

Para a análise das resoluções e respostas aos problemas no tocante aos níveis de compreensão do RP reportamo-nos à Tall e Vinner (1981, p.2), os quais definem imagem conceitual como a estrutura cognitiva total, construída na mente de uma pessoa a respeito de determinado conceito matemático, abrangendo todas as ideias, imagens mentais, impressões, representações visuais e descrições verbais relativas às propriedades e processos que envolvem aquele conceito. Segundo Tall e Vinner (1981, p. 2):

Como resultado e por meio de experiência de todos os tipos que uma pessoa se vê envolvida ao longo do tempo a imagem de um conceito vai se constituindo e se transformando continuamente quando ela passa pelo enfrentamento de novos estímulos [...].

A julgar pelos resultados conceituais (não o resultado numérico correto/errado) apresentados pelos sujeitos do estudo ao longo das resoluções dos problemas e levando em conta as considerações que foram feitas acima, consideramos ter havido um número muito pequeno de resoluções que se aproximaram do que Tall e Vinner (1981, p.2) consideram ocorrer em relação à imagem conceitual relativa à apropriação do RP, e a modelagem via estabelecimento de uma função linear.

Para este estudo, e mesmo considerando o pequeno universo de 12 sujeitos do estudo, consideramos que apenas os alunos A5, A7 e A9 podem ser enquadrados no perfil descrito na citação acima. À luz do que Tall e Vinner (1981, p.2) preconizam em relação à imagem de um conceito, parece-nos razoável e justa a justificativa de os resultados obtidos constituírem reflexo de uma experiência conceitual muito recente para os alunos, segundo a qual eles 
não tiveram suficiente tempo e diferenciadas experiências para fazerem a transformação da imagem do conceito que foi solicitado se apropriassem e exercitassem na resolução de problemas.

O Quadro 2, a seguir, mostra o desempenho de cada um dos sujeitos do estudo no tocante ao nível de solução empreendido para a resolução de cada um dos problemas constantes da Ficha de Avaliação. Para cada aluno é possível identificar o nível de resolução objeto do encaminhamento escolhido, bem como saber se a resolução resultou em resposta quantitativa correta (C) ou errada (E).

Quadro 2: Desempenho dos 12 sujeitos do estudo, por nível de
resolução, considerando resposta quantitativa correta(C) ou
errada(E), para todos os problemas da Ficha de Avaliação.

\begin{tabular}{|c|c|c|c|c|c|c|}
\hline Problema & Problema & Problema & Problema & Problema & Problema & Problema \\
\hline Aluno & 1 & 2 & 3 & 4 & 5 & 6 \\
\hline
\end{tabular}

\begin{tabular}{|l|l|l|l|l|l|l|}
\hline A1 & Nível 2 C & Nível 2 C & Nível 2 C & Nível 2 C & Nível 2 C & Nível 2 C \\
\hline A2 & Nível 1 C & Nível 1 C & Nível 1 C & Nível 1 C & Nível 1 C & Nível 1 C \\
\hline A3 & Nível 2 C & Nível 2 C & Nível 2 C & Nível 2 C & Nível 2 C & Nível 2 C \\
\hline A4 & Nível 2 C & Nível 2 C & Nível 2 C & Nível 2 E & Nível 2 C & Nível 2 C \\
\hline A5 & Nível 2 C & Nível 2 C & Nível 2 C & Nível 2 E & Nível 2 C & Nível 2 C \\
\hline A6 & Nível 2 E & Nível 1 E & Nível 1 E & Nível 1 C & Nível 1 E & Nível 1 E \\
\hline A7 & Nível 4 C & Nível 0 & Nível 0 & Nível 0 & Nível 6 C & Nível 5 C \\
\hline A8 & Nível 2 C & Nível 2 E & Nível 2 C & Nível 1 C & Nível 1 C & Nível 1 C \\
\hline A9 & Nível 3 C & Nível 4 C & Nível 6 C & Nível 6 C & Nível 6 C & Nível 5 C \\
\hline A10 & Nível 1 C & Nível 1 C & Nível 1 C & Nível 1 C & Nível 1 C & Nível 1 C \\
\hline A11 & Nível 2 C & Nível 2 C & Nível 2 E & Nível 2 C & Nível 2 C & Nível 1 E \\
\hline A12 & Nível 2 C & Nível 2 C & Nível 2 C & Nível 2 C & Nível 2 C & Nível 2 C \\
\hline
\end{tabular}

Fonte: Dados do estudo.

O Quadro 2, acima, mostra o desempenho de cada sujeito de estudo no tocante ao nível de resolução empreendido para a resolução de cada um dos problemas propostos na Ficha de Avaliação. Para cada problema e aluno é possível identificar o nível de resolução que foi objeto de escolha, para encaminhar a resolução, e se esta resultou em resposta quantitativa correta (C) ou errada (E). Pelo Quadro 2, verifica-se que os Problemas 1, 5 e 6 foram os únicos em que não houve um só aluno que não tenha tentado resolvê-los, isto é, não houve quem tenha deixado em branco o espaço para a resolução de cada um desses respectivos problemas. Também se verifica que o Problema 6 foi o único em que 2 sujeitos de estudo apresentaram resolução Nível 5.

Identifica-se que apenas os alunos A7 e A9 resolveram pelo menos um dos problemas por meio do exercício do RP. O exercício do RP, na íntegra, está indicado por meio de um grifo no Quadro 2, acima. Apenas os alunos A7 e A9 resolveram o problema 6 por meio do conceito de proporcionalidade, 
acompanhado de uma operação: multiplicação ou adição, configurando resolução de Nível 5. Também, que somente o aluno A5 mobilizou o RP na resolução do problema 6 via Nível 6 de resolução, e apenas o aluno A9 resolveu o problema 4 por meio do exercício do RP na íntegra (conforme a indicação na cor vermelha, no Quadro 2, acima). Importante notar que não houve qualquer menção ou indício de considerar proporcionalidade (Níveis 3 e 4 de resolução) nas resoluções que foram apresentadas para os 3 últimos problemas constantes da Ficha de Avaliação, por todos os 12 sujeitos do estudo. Ou seja, para os 3(três) últimos problemas em nenhuma das resoluções apresentadas o RP foi mobilizado, com grande prevalência de resoluções por meio de cálculos diretos ou a aplicação direta da "Regra de Três".

Tais fatos chamam-nos a atenção para a pouca ou nenhuma apropriação do conceito de proporcionalidade direta na resolução de problemas de aumentos ou decréscimos percentuais, pelos sujeitos do estudo. Identifica-se que apenas os sujeitos do estudo A5, A7 e A9 procuraram fazer uso do conceito de proporcionalidade por meio do exercício do RP, com o propósito de encaminhar a resolução e obter a resposta quantitativa. Também, que este mesmo procedimento ocorreu em até quatro dentre os seis problemas propostos e feito por poucos sujeitos do estudo. Ademais, chamou-nos atenção também o fato de o sujeito A7 ter deixado em branco o problema 4. Talvez seja um sinal do desconhecimento dos conceitos envolvidos ou razão que não pode ser identificada, uma vez que nos problemas 5 e 6 o rendimento deste sujeito do estudo foi muito bom, conforme mostrado no Quadro 2.

\section{Considerações finais}

Embora o estudo tivesse tido o propósito de apresentar alternativas diferenciadas de resolução para diferentes tipos de problemas associados com situações simples envolvendo o conceito de proporcionalidade e o cálculo de percentuais e valores, via modelos de funções lineares associadas, os resultados da avaliação apontam que talvez esse propósito não tenha sido percebido, compreendido, apropriado e aprendido, pela maioria dos sujeitos do estudo, durante a resolução dos problemas presentes na Ficha de Avaliação.

Ressalte-se que já durante os momentos de discussões e reflexões, também não foi grande a aceitação dos alunos por resoluções encaminhadas via métodos alternativos que não as que que recorriam a procedimentos de cálculos, em si, apenas. Por conta disso, já nestes momentos, em sua maioria os sujeitos do estudo preferiam fazer uso da Regra de Três a refletirem acerca de como um mesmo problema possa ser resolvido via o modelo de uma função linear.

Assim, não obstante o engajamento dos alunos durante a fase de desenvolvimento e exploração da proposta de alternativas de resolução, resultados da avaliação mostraram que as resoluções que foram apresentadas estão distantes daquilo que era esperado pelo pesquisador-professor quando da etapa de elaboração da proposta de estudo. Tal constatação é corroborada com a afirmação de Fischbein (1994, p. 232) a respeito de como um conteúdo pode ser explorado pelo professor. 
Por conta da flexibilidade permitida pela metodologia de poder apresentar e desenvolver o conteúdo proporcionalidade, no universo da Matemática Financeira, por meio da resolução de problemas, segundo o exercício do RP, e os objetivos iniciais que foram traçados, consideramos que a metodologia escolhida adequou-se bem às situações envolvidas, embora o estudo tivesse sido aplicado em pequena escala: pequeno número de alunos: variável, até o máximo de 28 alunos.

Nos momentos de desenvolvimento da proposta, e que antecederam a avaliação: exploração, reflexão, discussão de alternativas de resolução para os 13 problemas, nos grupos menores de até 4 alunos e no grupo como um todo, com a mediação do professor, o professor-pesquisador identificou certa rejeição dos sujeitos do estudo em relação aos procedimentos utilizados (modelagem, via funções lineares) e às considerações que iam sendo feitas, conforme foi mostrado para o exemplo de resolução do Problema 2, como acima.

Por vezes os alunos se manifestavam a favor do uso da "Regra de Três" como procedimento único de resolução para a situação envolvida, por considerála de "uso imediato" e "mais simples", nas palavras de alguns deles. Salientavam não haver necessidade de conhecerem outro procedimento de resolução uma vez que já dominavam um método mais prático, econômico e simples que dava conta de resolver.

O Problema 1 foi objeto de análise no artigo anterior e o Problema 2 neste trabalho. Os outros 11 problemas que foram objeto de reflexões e discussões acerca de resoluções alternativas seguiram procedimentos análogos aos que foram apresentados aqui, para o Problema 2. Importante mencionar que a escolha da estratégia de resolução para cada problema cabia decidir, exclusivamente, a cada aluno e/ou o grupo menor. Em seguida, para cada problema em discussão, o professor pedia a todos que refletissem como ele poderia ser resolvido por meio do exercício do RP via modelo de função linear.

Nas resoluções que foram apresentadas pelos sujeitos do estudo para os 6 problemas constantes da Ficha de Avaliação o RP foi mobilizado na íntegra apenas por 2 sujeitos de estudo: A7, no Problema 5 e A9, nos Problemas 3, 4 e 5. Já o conceito de proporcionalidade por meio do modelo de uma função linear não foi utilizado por nenhum dos sujeitos do estudo quando da resolução dos problemas constantes da Ficha de Avaliação.

O pesquisador refletiu acerca dos resultados obtidos pelos sujeitos do estudo via modelagem de funções lineares; sobre os tipos de resolução apresentados e em relação à comunicação das respostas, considerando que para todas essas questões o modo como o estudo se desenvolveu foi determinante para tal.

O pesquisador entendeu que tanto a escolha dos problemas constantes da Ficha de Avaliação quanto a maneira como o estudo foi encaminhado e o tempo destinado ao estudo possivelmente não tenham sido interessantes e suficientes para despertar o interesse por soluções alternativas. Também, não suficientes para motivar os alunos em relação à alavancar conhecimentos relacionados ao estudo das funções lineares e o conhecimento formal em relação ao 
estabelecimento da "Regra de Três" como um caso particular de combinação de propriedades para este tipo de funções do $1^{\circ} \mathrm{grau}$. Ademais, pelo fato de todos os problemas propostos na Ficha de Avaliação permitirem a quem se propõe resolvê-los não haver necessidade imperiosa de ter de lançar mão do exercício do RP (diretamente ou não) para encaminhar a resolução, e obter a solução em seguida.

Ou seja, os problemas que foram propostos garantiam aos sujeitos do estudo liberdade para encaminhar a resolução que consideravam mais rápido e seguro, como a Regra de Três (que eles já conheciam de experiências anteriores e foi largamente utilizada), por exemplo, segundo procedimentos que não exigiam o exercício do RP.

Sendo assim, mesmo considerando que houve bom envolvimento dos alunos no estudo durante as aulas que antecederam a avaliação por meio das discussões encaminhadas (tanto nos grupos menores quanto no grupo maior como um todo), não houve qualquer manifestação explícita de aprovação e aceitação para a proposta do estudo por parte dos 12 sujeitos do estudo que entregaram a Ficha de Avaliação.

O pesquisador identificou que parte considerável do quantitativo de alunos da turma - considerando a totalidade de 28 alunos - ainda é reticente a mudanças metodológicas abruptas, que fogem do ensino habitual. Ou seja, ainda é forte o desejo dos alunos de não se arriscarem em resolver problemas de modo diferente daquele que já estão acostumados fazer.

Sendo assim, uma vez conhecido um procedimento de aprendizagem ensino sobre o qual os conhecimentos acerca de um particular conteúdo - que já foram por eles apropriados, e que já estão fortemente consolidados - e se esse procedimento atende às necessidades particulares de momento, para a resolução de determinados tipos de problemas, isso é o bastante para não fazer novos experimentos. Isto é, se o procedimento já está de bom tamanho, não há razões para que façam novos experimentos e mudanças.

Entendemos ser importante e necessário que os alunos aprendam Matemática com a compreensão de conceitos, significados e procedimentos, e por conta disso salientamos tal fato aqui e durante todo o estudo. Em Matemática Financeira tal consideração não é diferente Assim, aproveitamos para apontar o excesso de procedimentos algorítmicos que se identifica nas salas de aula os quais devem dar lugar a um ensino que priorize a disseminação de conceitos matemáticos e a apropriação destes e de seus significados, para a aplicação dos conhecimentos produzidos, em diferentes contextos.

Por conta da proposta de ensino aprendizagem relativamente ao exercício do RP via modelos de funções lineares, o autor deste trabalho espera que a aplicação do conceito de proporcionalidade e o exercício do RP - como feito ao longo do estudo - seja lembrado pelos alunos, mais adiante, e não apenas durante a resolução de problemas de Matemática Financeira, ainda no Ensino Médio, mas em outros contextos, como em Física, por exemplo. 
O autor aproveita para (re) afirmar que cabe ao professor de Matemática promover a construção de novos conhecimentos, em processo dialógico constante e de modo pró ativo a partir dos conhecimentos prévios dos alunos, e das experiências que eles trazem para a escola.

Entendemos que as ações cognitivas tornam-se pobres (sem valor) e com o tempo ficam empobrecidas mais ainda, à medida que o aluno recebe informações prontas e não é instigado pelo professor a construir conhecimentos, pois o conhecimento não é transferível mas precisa, sim, ser construído.

As informações que via de regra tem se tornado corriqueiras na escola (por exemplo, do tipo "É assim que se faz"; "Faça isso"; "Faça assim"; "Não, não faça assim que vai dar certo"; "Lembra daquilo que eu falei para vocês?"; "Lembra de como eu fiz no quadro branco, faça igual") precisam ser banidas do ensino, imediatamente.

Para tais orientações aos alunos em sala de aula, em seguida, via de regra, aplicam-se informações e procedimentos de "como fazer", "como proceder", "como vai dar certo" em exercícios escolares repetitivos do tipo "calcule; determine, ache, etc e deixa-se de explorar o conceito e seus significados. Em resumo, o ensino prioriza ações do tipo "siga o modelo".

Entendemos que a ação cognitiva só se materializa quando o estudante coloca algo novo em relação ao que já conhece, e o que vivenciou, e assim ele avança nos conhecimentos e saberes após ultrapassar desafios importantes (e necessários), próprios dos momentos de ensino aprendizagem.

Se o aluno faz algo sem pensar tal qual o seu professor fez é sinal de que sabe imitar bem o que lhe apresentaram, mas talvez desconheça acerca do que trata o conceito vez que não se apropriou dele.

Portanto, tem-se alunos que não dominam o conceito para utilizá-lo em outros contextos. Sendo assim, fica difícil aprender por imitação. Não verdade não aprende mas acostuma-se a reproduzir procedimentos.

Por outro lado, é possível que um aluno automatize certo procedimento por conta de seu recorrente uso em problemas similares - o que constitui uma habilidade matemática. Mas ela é empobrecida por conta de ele desconhecer as razões conceituais que permitem o seu uso ali e, talvez, não acolá.

\section{REFERÊNCIAS}

COBB, Paul; CONFREY, Jere; DiSESSA, Andrea; LEHRER, Richard; SCHAUBLE, Leona. Design Experiments in Educational Research. American Educational Research Association, v. 32, n.1,jan/fev, p.9-13, 2003.

FRANCO, Maria Amélia Santoro. Pedagogia da Pesquisa-ação. Educação e Pesquisa, São Paulo SP, v. 31, n. 3, set/dez, p.483-502, 2005.

Disponível em: https://www.scieo.br/pdf/ep/v31/n3/a11v31n3.pdf. Acesso em: 19 dez. 2019. 
FISCHBEIN, Efrain. The interaction between the formal, the algorithmic and the intuitive components in a mathematical activity. In: Didactics of Mathematics as a Scientific Discipline. Mathematics Education Library. Dordrecht: Kluwer Academic Publishers, 1994.

FREIRE, Paulo. Pedagogia da autonomia: saberes necessários à prática educativa. São Paulo: Paz e Terra, 2013.

LIMA, Elon Lages. Grandezas Proporcionais. In: Meu Professor de Matemática e outras histórias. Rio de Janeiro. RJ: IMPA Instituto de Matemática Pura e Aplicada. VITAE Apoio à Cultura, Educação e Promoção Social, p.125-141, 1991.

TEIXEIRA, Paulo Jorge Magalhães. Análise da produção de alunos do 70 ano do Ensino Fundamental, veiculada em cartazes, acerca da presença ou não de proporcionalidade em situações cotidianas de preços de produtos. TANGRAM

- Revista de Educação Matemática, v.3, n.2, p.110-137, 2020a.

TEIXEIRA, Paulo Jorge Magalhães. Mobilizando o raciocínio proporcional na resolução de problemas de percentual. REVEMAT - Revista Eletrônica de Educação Matemática. v.15, p.01-21, jan/dez, 2020b.

SHULMAN, Lee $S$. Those who understand: knowledge growth in teaching. Educational Researcher, v.15, n.2, p.4-14, 1986.

TALL, David e VINNER, Shlomo. Concept image and concept definition in mathematics with particular reference to limits and continuity. Educational Studies in Mathematics, v.12, p. 151-169, 1981.

Recebido em: 22 de agosto de 2020. Aceito em: 30 de novembro de 2020 . Publicado em: 30 de junho de 2021. 